\title{
Passive Micromixer for Luminol-peroxide Chemiluminescence Detection
}

\author{
Khoi Seng Lok ${ }^{1}$ Yien Chian Kwok ${ }^{1}$ and Nam-Trung Nguyen ${ }^{2}$
}

${ }^{1}$ National Institute of Education, Nanyang Technological University, 1 Nanyang Walk, Singapore 637616, Singapore.E-mail: yienchian.kwok@nie.edu.sg

${ }^{2}$ School of Mechanical and Aerospace Engineering, Nanyang Technological University, 50 Nanyang Avenue, Singapore 639798, Singapore.E-mail: mntnguyen@ntu.edu.sg

Key words: passive micromixer, staggered herringbone, chemiluminescence, luminol, cobalt catalysis, hydrogen peroxide

\begin{abstract}
s
This paper reports a microchip with an integrated passive micromixer based on chaotic advection. The micromixer with staggered herringbone structures was used for luminol-peroxide chemiluminescence detection. The micromixer was examined to assess its suitability for chemiluminescence reaction. The relationship between the flow rate and the location of maximum chemiluminescence intensity was investigated. The light intensity was detected using an optical fiber attached along the mixing channel and a photon detector. A linear correlation between chemiluminescence intensity and the concentration of cobalt (II) ions or hydrogen peroxide was observed. This microchip has a potential application in environmental monitoring for industries involved heavy metals and in medical diagnostics.
\end{abstract}

\section{Introduction}

Micromixers can be integarted in Lab-on-Chip (LoC) applications to improve mixing in laminar flow regime. Improved mixing can be achieved actively with external disturbance or passively with chaotic advection at high flow rate. A high flow rate also results in a shorter residence time and a faster analytical time. As mentioned above, micromixers are categorized as active and passive types. ${ }^{1,2}$ Passive 
micromixers relies on modifications in the shape or the geometry of the mixing channel. Due to the lack of active components, passive mixers are relatively easy to implement in a LoC system. Most passive micromixers use chaotic advection to improve mixing. ${ }^{3-8}$ Stroock et al. introduced the staggered herringbone mixer (SHM) based on chaotic advection. ${ }^{6,9}$ Following this work, the channel designs were modified in various manners to improve mixing performance. ${ }^{7,10}$ Mei et al. showed that having the herringbone pattern in the mixing channel improved the sensitivity of luciferase assay by three times. ${ }^{11}$

Simple Y-shaped or T-shaped micromixers were often used in LoC application. Wei et al. reported a simple mixer for the determination of benzoyl peroxide in flour. ${ }^{12} \mathrm{He}$ et al. developed a LoC for micro flow injection analysis ( $\mu$ FLA) to determine the amount of nitrite in food. ${ }^{13}$ Marle and Greeway used a LoC to determine the amount of hydrogen peroxide in rainwater. ${ }^{14}$ These devices have a simple serpentine microchannel as a mixing channel. Since simple T-mixers rely on molecular diffusion for mixing, the flow rate is limited to about 20 or $50 \mu 1 \mathrm{~min}^{-1}$ and cannot be increased further. Based on Einstein-Smoluchowski diffusion theory, the time estimated for a protein with a diffusion coefficient of $D$ $\approx 10^{-6} \mathrm{~cm}^{2} \mathrm{~s}^{-1}$ to move across half of a channel with a width of $1 \mathrm{~mm}$ is about 125 seconds. To warrant a residence time less than 125 seconds, a low flow rate is required for the operation of these devices.

Micromixers often serve as a microreactor. Tan et al. used a modified SHM for detection of nerve agent sarin in blood. ${ }^{15}$ The modified SHM had been used as a reactor for nerve gas regeneration. Potassium fluoride was mixed with sarin-spiked whole blood to regenerate sarin for further detection. The optimized flow rate for this reactor was $20 \mu \mathrm{min}^{-1}$, because the required time for enzymatic reaction was considered. Xu et al. also used a SHM for glucose detection. ${ }^{16}$ Although, the SHM was included to assist mixing, a low flow rate was used. Luminol and hexacyanoferrate was injected at $10 \mu 1 \mathrm{~min}^{-1}$ into a 32-mm long SHM. Subsequently, glucose was injected at $25 \mu 1 \mathrm{~min}^{-1}$ into a $35-\mathrm{mm}$ long reactor with of immobilized particles for glucose oxidase. The reactants were mixed in another $34.7-\mathrm{mm}$ long SHM. When the sample flow rate increased from 10 to $70 \mu 1 \mathrm{~min}^{-1}$, the detected signal decreased, apparently due to insufficient reaction time as claimed by the author. According to Lin's work, ${ }^{10}$ a SHM requires a 
mixing length of $60 \mathrm{~mm}$ to achieve a standard a deviation of uniformity of 0.05 . Hence, the length of a SHM warrants the sufficient residence time and proper mixing at higher flow rates.

Therefore, introduction of appropriate micromixer design, as a microreactor in the LoC, may facilitate maximum mixing efficiency, followed by maximum reaction efficiency at a higher flow rate setting. But, the flow rate is limited by the enzymatic reaction time in the respective chemical system. There are very few reports of implementing the passive micromixer into LOC device; fewer put an intensive study on the micromixer design to implement it correctly.

Chemiluminescence (CL) detection method is appreciated in LoC application. It does not require an excitation light source and gives lower background interferences. But the CL intensity diminishes rapidly when the reactants are mixed. Hence, the detector must be readily available to capture the light signal once the reaction is initiated. There are many CL systems such as luminol, acridinium compounds, coelenterazine, dioxetances and luciferase with various applications in immunoassays, receptor assays, DNA probes, biosensor and more. ${ }^{17}$ Luminol (5-aminophthalhydrazide) CL is a well-characterized reaction system that have been useful for various applications such as monitoring of metals and other pollutants in water, ${ }^{18,19}$ immunoassays and DNA analysis,${ }^{20}$ dating of human remains ${ }^{21}$ and more. During the reaction, luminol is oxidized to 3 -aminophthalate ions with the aid of a catalyst or co-oxidant under alkaline aqueous conditions, producing water, nitrogen gas and light (wavelength $=425 \mathrm{~nm}$ ). In this study, the cobalt catalysis of luminol-peroxide CL was used. ${ }^{22}$

Marle and Greenway developed a glass microchip for analysis of hydrogen peroxide in rain water using a luminol-peroxide CL system. ${ }^{14}$ The sensitive 8 -mm aperture sized photon detector was aligned to a rectangular channel network, capturing all unmixed, mixing, reacting and reacted reagents. As mentioned earlier, the microchannel did not feature micromixer design. A parabolic curve was fitted from 0.1 to $7.5 \mu M$. The linearity of the signal response was limited at 0.1 to $1 \mu M$. Compared this work to the pioneered work in 1975 by Burdo and Seitz, ${ }^{22}$ the maximum CL intensity was between $1 \mathrm{mM}$ to $2 \mathrm{mM}$, 
and linearity was observed for lower concentration of $\mathrm{H}_{2} \mathrm{O}_{2}$ below $1 \mathrm{~m} M$. This discrepancy can be explained by the lack of mixer design in the microchip. However, miniaturization of benchtop laboratory work to $\mu$ TAS should be comparable.

In this preliminary study, we investigated the dependency of flow rate and mixing channel length on CL intensity in a chaotic advection passive micromixer. Our ultimate objective was to develop a CL microchip system suitable for determination of low concentration of analyte with shorter analytical time. This micromixer design was adopted from Tan et al.'s work ${ }^{15}$. It had been used for mixing of reagents for enzymatic reaction. But, it had not been characterised in CL related detection. A low flow rate had to be used to cater for the enzymatic reaction time. Since the micromixer made use of chaotic advection, higher flow rate should result in better mixing. Hence, its potential was not utilised. A long reaction channel was created in our microchip to ensure that the optimum micromixer mixing channel length and maximum CL intensity could be determined. An optical fiber connected to a photon detector was used to probe the pinpoint CL light intensity along the channel. The optimum micromixer mixing channel length might be determined from using this method. After the optimisation of the flow rate and mixing channel length, the microchip was used to demonstrate the determination of cobalt (II) ions and hydrogen peroxide. The luminol CL intensity followed good correlation with increasing cobalt (II) ions and hydrogen peroxide at low concentrations. For future microfluidic devices, the appropriate length of passive micromixer, accompanied by shorter analytical time could be fabricated.

\section{Materials and Methods}

\section{Microchip Design}

The micromixer design was adopted from Tan et al.'s work. ${ }^{15}$ The micromixer acts as the reaction channel, which is $1.8 \mathrm{~mm}$ in wide and $0.4 \mathrm{~mm}$ high. The staggered herringbone microstructures are designed as pleats with a height of $0.2 \mathrm{~mm}$ and width of $0.5 \mathrm{~mm}$. The height of the pleats is exactly half of the channel's depth. Unlike the traditional SHM design, this micromixer has two unique features. First, 
the channel is serpentine. This can reduce the foot-print of the microchip. The bending at each corner twists the interface between the two fluids at each junction, and thus further promotes chaotic advection. Second, each period of the channel consists of forward and reversed staggered herringbone grooves. This reverses the direction of the secondary flow in each period to improve the folding effect on each half of the channel, allowing better catalytic interaction between reactants. The reaction channel has a total length of $202 \mathrm{~mm}$. The estimated total channel volume is $145 \mu 1$. The length of the channel from "entrance" to "middle" is $111 \mathrm{~mm}$. Figure 1 shows the schematic layout of the microchip.

\section{Microfabrication}

CorelDraw was purchased from Corel Co. (Canada). A commercial $\mathrm{CO}_{2}$ laser system, Universal M300 Laser Platform, was obtained from Universal Laser Systems Inc. (Arizona, USA). Polymethyl methacrylate (PMMA) sheet of $1 \mathrm{~mm}$ thickness was obtained from Ying Wang Acrylic Trading (Singapore). CorelDraw was used to design the pattern required. The pattern was then transferred the $\mathrm{CO}_{2}$ laser system to make the PMMA parts. The parts were aligned and thermally bonded at low pressure, $170^{\circ} \mathrm{C}$. Tube plugs were glued by epoxy glue to provide access to the inlets and outlet to the microchip.

\section{Chemiluminscence reagents}

All reagents were analytical grade. All salts were dissolved in ultrapure water $\left(18.2 \mathrm{M} \Omega \mathrm{cm}^{-1}\right)$ and filtered. Luminol was obtained from Fluka (Gillingham, Dorset, UK). Sodium carbonate $\left(\mathrm{NaHCO}_{3}\right)$ and cobalt(II) nitrate $\left(\mathrm{Co}\left(\mathrm{NO}_{3}\right)_{2} \cdot 6 \mathrm{H}_{2} \mathrm{O}\right)$ was obtained from Ajax Finechem (Australia). Sodium bicarbonate anhydrous $\left(\mathrm{Na}_{2} \mathrm{CO}_{3}\right)$ was obtained from GCE Laboratory Chemicals (Germany). 30\% (v/v) hydrogen peroxide $\left(\mathrm{H}_{2} \mathrm{O}_{2}\right)$ was obtained from Scharlau (Australia).

Carbonate buffer solution was adjusted to $\mathrm{pH} 10.0$ by mixing $0.1 \mathrm{M} \mathrm{NaHCO}_{3}$ and $0.2 \mathrm{M} \mathrm{Na}_{2} \mathrm{CO}_{3}$ dissolved in deionised water. Luminol was dissolved in this $\mathrm{pH} 10$ buffer solution at $65 \mathrm{mg}$ per $250 \mathrm{ml}$ and filtered with disposable syringe filter $(0.22 \mu \mathrm{m})$, giving a concentration of $1 \mathrm{mM}$. A stock solution of $1 \mathrm{mM}$ cobalt(II) nitrate in deionised water was made, and diluted in deionised water accordingly for use. 
$30 \%(\mathrm{v} / \mathrm{v})$ hydrogen peroxide was diluted to $3 \%(\mathrm{v} / \mathrm{v})$ in this experiment in deionised water and kept in ice before use. All reagents were prepared fresh before the experiments.

\section{Experimental Setup}

A programmable syringe pump, Kd Scientific KDS 250 was purchased from Microdialysis Infusion Pump (Massachusetts, US). H7467 photon detector with a microcontroller and RS-232C interface was obtained from Hamamatsu (Japan). Analysis 3.4 was obtained from Veriner Software.

A mixture (1:1) of diluted hydrogen peroxide and cobalt(II) solution and luminol in carbonate buffer were introduced by $5 \mathrm{ml}$ disposable syringes through the syringe pump via Teflon tubing into the microchip. The flow rate in the main channel is twice the flow rate in the inlet. CL light intensity was captured by attaching a $1 \mathrm{~mm}$ optical fiber from the chip to the adaptor of the photon detector. This experimental setup is shown in Figure 2.

This photon detector was interfaced directly to a personal computer via serial connection and data was directly captured by self-written Q-basic software at $30 \mathrm{~ms}$ or $50 \mathrm{~ms}$ integration time. The data was obtained over a $10 \mathrm{~s}$ interval for each reading. The data points were exported to the software, Analysis 3.4 for further analysis, and graphs were then plotted using Excel 2007. The counts numbers was averaged and converted to per ms interval. All the experiments and readings were conducted in a dark room to eliminate background readings. Each set of experiments were repeated more than 5 times for reliable data. Outliers were removed from the analysis.

\section{Results and Discussions}

\section{Effect of flow rate on $C L$ intensity}

To investigate the effect of the flow rate on the CL intensity emitted from the reactions, an optical fiber was attached at a fixed position of $131 \mathrm{~mm}$ from the entrance on the microchannel. A mixture $(1: 1)$ of $10^{-5} M$ cobalt(II) ions and $3 \%(\mathrm{v} / \mathrm{v}) \mathrm{H}_{2} \mathrm{O}_{2}$, and luminol in carbonate buffer were introduced steadily by 
mechanical syringe pump into the microchip, Figure 2. This created a continuous flow system, producing a constant luminescent light in the microchannel. The flow rates of the main channel were set progressively ranging from 1.67 to $16.7 \mu 1 \mathrm{~min}^{-1}$. Given that the estimated total channel volume was $145 \mu$, the corresponding minimum analytical time was 1.45 hours to 8.72 minutes. The entrance of the microreactor was taken to be the centre of the T-junction when the fluids first contact each other, Figure 1. Light emitted from the CL reaction was captured by a flexible, detachable optical fiber and lead to the photon detector. Collected data was analysed in the computer.

Figure 3 shows the effect of varying flow rate in the channel on CL intensity emitted from the reactions. A linear correlation was observed between flow rate and CL intensity, $\mathrm{R}^{2}=0.992$. The general trend is CL intensity emitted from the equivalent concentration of reactants increases with increased flow rate.

There are several explanations for this phenomenon. A straightforward answer is more reagents are pumped into the microchip at a higher flow rate and therefore a brighter signal is observed. Another, the micromixer makes use of the mixing principle of chaotic advection. ${ }^{15}$ The unique feature about such a micromixer is, a higher flow rate will result in the generation of faster vortices, in turn, a more vigorous mixing. ${ }^{1}$ Therefore, more reactants can interact with a more dynamic flow.

A high flow rate setting is recommended to give a brighter CL signal. This will also reduce the analytical time required for each sample. This is done at the cost of excessive use of reagents. An increased in flow rate will also increases the pressure within the microchannel in the microchip. This may lead to leakage. In usual cases, an extreme high flow resistance in the channel may cause the mechanical syringe pump to stall. Hence, a compromise between high signal magnitude and leakage needs to be found for an optimal flow rate.

\section{Effect of mixing channel length on CL intensity}


Next, the effect of mixing length on CL intensity emitted from the reactions was explored for flow rates ranging from 1.67 to $16.7 \mu 1 \mathrm{~min}^{-1}$. Using the similar experimental conditions as described previously, the optical fiber was attached at various locations on the chip to measure this light signal. The optical fiber was able to provide pin-point information on the CL reaction along the channel. This enabled us to understand how vigorous the reaction was at that particular point and determine the location of maximum CL intensity along the channel.

Figure 4 shows the CL intensity emitted from the reactions captured along the mixing channel length for the different flow rate. For each flow rate, the CL intensity from the equivalent concentration of reactants increased, and then decreased along the channel. The maximum CL intensity occurred at around $100 \mathrm{~mm}$ along the channel. A possible explanation for this is that the batch of reagents that is being introduced into the microchip is not well-homogenized initially. Chemical reactions occur between the molecules is restricted to the portion of molecules that happens to be mixed, and interacts with each other. When the reactants are more homogenized later after proper mixing, maximum interactions between molecules occur, maximum number of reactions happens and photon emission is at the maximum. This $\mathrm{CL}$ intensity from the reactions drops as the batch of reagents is exhausted later along the channel. As the reaction proceeds over time, these reactants are diminished, the number of reactions reduces, and the amount of photons emitted from the reaction decreases. Hence CL intensity also drops along the channel.

The trendlines were curve-fitted in graph, Figure 4. Using differential calculus, the derivative gradient of the trendline was obtained for each flow rate from 1.67 to $16.7 \mu 1 \mathrm{~min}^{-1}$ in the channel. From these derivatives, the position of the maximum CL intensity located on the channel was calculated. The flow rate in the channel was converted to velocity by removing the cross-sectional area of the channel. The velocity in the channel was plotted against the position of maximum CL intensity on the channel, Figure 5. A linear trend was observed for the position of the maximum CL intensity versus the velocity. The first-order fitting of this relationship is $\mathrm{L}=27.1 v+99.4$, where $\mathrm{L}$ is the position of the maximum $\mathrm{CL}$ intensity and $v$ is the velocity of the fluid flow in the channel, $\mathrm{R}^{2}=0.986$. The gradient constant, $27.1 \mathrm{~s}$, 
represented the reaction time needed for the delay CL reaction to reach the maximum CL intensity in the microchip system. This reaction time encompasses reaction kinetics and the effect of fluid mixing. A minimum length of $99.4 \mathrm{~mm}$ is necessary for the reagent to mix, react and emit the most intense CL light.

The detail of the effectiveness of this micromixer was investigated in our laboratory but had not been reported. Early studies indicated that the micromixer required a mixing length of $56 \mathrm{~mm}$ to achieve mixing efficiency of $90 \%$ at $20 \mu \mathrm{min}^{-1}$. This served a good mixing function in our application. Maximum CL intensity was postulated to be occurred approximately in that position from the entrance in the microchip initially. To our surprise, an extra length of $43.4 \mathrm{~mm}$ was needed to encounter for the delay in CL emission.

The oxidation of luminol in the CL reaction is a multiple step reaction. ${ }^{22,23}$ Several events may occur in the microreactor progressively. First, the different species of molecules: cobalt (II) ions, hydrogen peroxide and luminol get mix together. Then, they react by the formation of cobalt-peroxide complex, follow by the oxidation of luminol by one electron to a radical by the cobalt-peroxide complex. ${ }^{22}$ And finally, luminol radicals then react with hydrogen peroxide to produce the light emission in several steps reaction.

Therefore, the length of micromixer can be shortened to $60 \mathrm{~mm}$ to provide the need for mixing. And the reaction channel can be lengthened by $40 \mathrm{~mm}$ to cater for the relay in $\mathrm{CL}$ emission. The detector needs to be placed away from the entrance at $100 \mathrm{~mm}$. This is important because the fabrication of intricate microstructures in the microchannel can be time-consuming. These adjustments in the design will aid in the fabrication of future microchip, because it will reduce redundant features.

Using this relationship obtained earlier, the location of the maximum CL intensity along the mixing channel can be adjusted. For instance, to align the maximum CL intensity to the centre of the mixing channel at $110 \mathrm{~mm}$, the velocity in the channel is estimated to be $0.390 \mathrm{~mm} \mathrm{~s}^{-1}$, corresponding to a flow rate of $16.8 \mu 1 \mathrm{~min}^{-1}$ was chosen. The optical fiber records the intensity of the light signal at pinpoint 
location. Hence, it is important to know the precise location to attach optical fiber so that the capture of maximum CL intensity will not be missed.

\section{Effect of concentrations of cobalt(II) ions on CL intensity}

Next, the effects of the concentrations of cobalt(II) ions on CL intensity emitted from the reactions was investigated. The concentration of cobalt(II) ions that was being determined, was from $10^{-10}$ to $10^{-3}$ M. A mixture (1:1) of cobalt(II) ions and 3\% (v/v) $\mathrm{H}_{2} \mathrm{O}_{2}$, and $1 \mathrm{mM}$ luminol in carbonate buffer were introduced into the microchip. The flow rate was set to be $16.8 \mu 1 \mathrm{~min}^{-1}$. The minimum analytical time for each sample was 8.65 minutes. The optical fiber was attached to the microchip at $110 \mathrm{~mm}$ from the entrance.

The calibration graph for cobalt(II) ions against CL intensity was plotted, Figure 6 . The CL intensity increased with increasing concentration of cobalt(II) ions. The linear range was observed from $10^{-10}$ to $10^{-}$ ${ }^{3} M$. The fitting function is $\mathrm{y}=1680 \mathrm{x}+155, \mathrm{R}^{2}=0.998$, where $\mathrm{y}$ and $\mathrm{x}$ represent the CL intensity in counts and the concentration of cobalt(II) ions in $\mathrm{mM}, \mathrm{R}^{2}=0.995$. Deionised water was used in place of cobalt(II) ions, which gave a background (blank) of $0.24 \pm 0.28$.

The limit-of-detection (lod) was calculated from the $y$-intercept plus three times of standard deviation of the regression $(\mathrm{SD} x / \mathrm{y})$. The calculated lod was $1.06 \mathrm{n} M$. The differences in the number of counts in CL intensity between $10^{-10}$ to $10^{-5} \mathrm{M}$ were insignificant to produce appreciable differences for the measurement of cobalt(II) ions concentration. Hence, the useful linear range of the microchip would be $10^{-5}$ to $10^{-3} \mathrm{M}$.

Luminol CL reaction can be catalysed by various metal ions. Cobalt (II) ions are known to be more effective catalysts for this reaction compare to copper(II), nickel(II), iron(III), iron(II), manganese(II). ${ }^{24}$ The applications of the microchip would also be useful for the measurement of the water quality for the presence of heavy metals such as cadmium(II) and other ions. ${ }^{25}$ Assessing the level of heavy metals 
contamination is important to monitor pollution from industrial wastewater, safety of food, including agricultural and fishery products in marine coastal water. ${ }^{26}$

\section{Effect of concentrations of hydrogen peroxide on $C L$ intensity}

Hydrogen peroxide determination was another important application of luminol CL system. ${ }^{27}$ The CL intensity given off by different concentration of hydrogen peroxide from the reactions was studied. The concentration of cobalt (II) ions was fixed at $10^{-5} \mathrm{M}$.

The effect of the concentration of hydrogen peroxide from $1.47 \times 10^{-7}$ to $1.47 \times 10^{-1} \mathrm{M}$ on CL intensity was explored. From $1.47 \times 10^{-6}$ to $2.9 \times 10^{-4} \mathrm{M}$ of hydrogen peroxide, CL intensity of the reaction increased linearly with increasing concentration of hydrogen peroxide, Figure 7. The observed linear response was similar to the pioneered work by Burdo and Seitz. ${ }^{22}$ The equation of the line was $\mathrm{y}=29600 \mathrm{x}+8.28$, where $\mathrm{y}$ is the CL intensity in counts, and $\mathrm{x}$ is the concentration of hydrogen peroxide in $\mathrm{mM}, \mathrm{R}^{2}=0.999$. The background (blank) for this experiment was determined by deionised water in place of $\mathrm{H}_{2} \mathrm{O}_{2}$. The value was $8.28 \pm 0.72$.

The limit-of-detection (lod) was calculated from concentration of analyte required to give a signal equal to the background (blank) plus three times the standard deviation of the blank. The calculated lod was $73.1 \mathrm{nM}$. At $1.47 \times 10^{-7} \mathrm{M}$ of hydrogen peroxide, the CL signal was nearly indistinguishable from the background. Hence, the lowest reliable limit for determining hydrogen peroxide was $1.47 \mu M$ for this system.

This linear correlation implies that this microchip can be used for the determination of the concentration of hydrogen peroxide. Hydrogen peroxide is produced in oxidative stress in cells. It is a product from the oxidation of glucose, cholesterol and uric acid by oxidase enzyme. ${ }^{28}$ Hence, the detection of hydrogen peroxide is useful for medical diagnostics. Hydrogen peroxide can also be used to monitor rainwater, which is an efficient oxidiser of sulfur dioxide to produce sulfuric acid, an important compound in acid rain formation. ${ }^{14}$ 


\section{Further optimization of CL intensity in the microchip}

The pinpoint measurement using the optical fiber is only able to sample a small amount of reactant volume in the microchannel. Therefore, the CL intensity captured from the reaction was weak. Higher flow rate set will increase the CL intensity. Given a flow rate of $100 \mu 1 \mathrm{~min}^{-1}$, the CL intensity at the position of $131 \mathrm{~mm}$ from the inlet is about 1670 counts for the concentration of $10^{-5} \mathrm{M}$ cobalt(II) ions. The analytical time for each sample was about $1.5 \mathrm{~min}$, which is a relatively short time. However, this high flow rate setting is limited by the amount of analytical sample, bonding strength of the microchip, and fitting of the connector tubing.

The CL intensity emitted from the reaction can be increased by increasing the reactant volume for a fixed concentration. To put on trial, the optical fiber is attached to the plug outlet on the microchip for the measurement of CL intensity emitted. The plug outlet holds an approximate volume of $0.5 \mathrm{~mm}^{3}$. The flow rate was $163 \mu 1 \mathrm{~min}^{-1}$ in the main channel. The analytical time for each sample was further reduced to about $1 \mathrm{~min}$. This flow rate will push the maximum CL intensity to the end of the reaction channel. A linear range of $10^{-9}$ to $10^{-5} \mathrm{M}$ was obtained between the concentration of cobalt(II) ions and CL intensity, following the fitting line $y=19450 x+89.1$, where $y$ and $x$ represent the CL intensity in counts and the concentration of cobalt(II) ions in $\mathrm{mM}, \mathrm{R}^{2}=0.995$. Based on the line gradients, this method can be 11 times more sensitive than attaching the optical fiber at $110 \mathrm{~mm}$. Hence, future work should aim to enlarge this reactant volume to expose more CL light to the photon detector. The detection limit and sensitivity may be lowered further by increasing the sampling reactant volume. Such microchip design may include a shorter micromixer and spiral channel detection system. ${ }^{11}$

\section{Conclusion}

A microchip, containing a passive micromixer as the reaction channel was characterized for luminolperoxide chemiluminescence (CL) detection. A high flow rate was found to lead to a brighter CL signal intensity and shorter analytical time. A parabolic distribution of CL intensity was found along the reaction channel. The maximum CL intensity of this distribution shifted with increasing flow rate. The 
concentration of cobalt ions or hydrogen peroxide correlated with CL intensity emitted from the reactions. Larger reactant volume was important for a more intense CL signal to increase its sensitivity. This approach seemed to be good for creating microchip for CL applications.

\section{References}

1. N. T. Nguyen and Z. Wu, Journal of Micromechanics and Microengineering 2005, 15.

2. V. Hessel, H. Löwe and F. Schönfeld, Chemical Engineering Science, 2005, 60, 2479-2501.

3. A. A. S. Bhagat, E. T. K. Peterson and I. Papautsky, Journal of Micromechanics and Microengineering, 2007, 17, 1017.

4. S. S. Hsieh and Y. C. Huang, Journal of Micromechanics and Microengineering, 2008, 18, 065017.

5. Y. C. Lin, Y. C. Chung and C. Y. Wu, Biomed Microdevices, 2007, 9, 215-221.

6. A. D. Stroock and G. M. Whitesides, Accounts of Chemical Research, 2003, 36, 597-604.

7. Y. J. Yang, H. H. Liao, K. H. Huang, Y. Y. Huang, C. W. Lin, L. J. Yang, F. S. Jaw, P. Z. Chang and C. K. Lee, Novel Designs of Herringbone Chaotic Mixers, 2006.

8. T. G. Kang, M. K. Singh, P. D. Anderson and H. E. H. Meijer, Microfluidics and Nanofluidics, 2009.

9. A. D. Stroock, S. K. W. Dertinger, A. Ajdari, I. Mezic, H. A. Stone and G. M. Whitesides, Science, 2002, 295, 647-651.

10. W. Lin, Chemical Engineering \& Technology, 2008, 31, 1210.

11. Q. Mei, Z. Xia, F. Xu, S. A. Soper and Z. H. Fan, Analytical Chemistry, 2008, 80, 6045-6050.

12. L. Wei, Z. Zhujun and Y. Liu, Food Chemistry, 2006, 95, 693-698.

13. D. He, Z. Zhang, Y. Huang and Y. Hu, Food Chemistry, 2007, 101, 667-672.

14. L. Marle and G. M. Greenway, Analytica Chimica Acta, 2005, 548, 20-25.

15. H. Y. Tan, W. K. Loke, Y. T. Tan and N.-T. Nguyen, Lab on a Chip, 2008, 8, 885-891.

16. Z. R. Xu and Z. L. Fang, Analytica Chimica Acta, 2004, 507, 129-135. 
17. C. Dodeigne, L. Thunus and R. Lejeune, Talanta, 2000, 51, 415-439.

18. X. Liu, A. Li, B. Zhou and C. Q. H. Ren, Chem Cent J., 2009, 3.

19. S. J. Ussher, A. Milne, W. M. Landing, K. Attiq-ur-Rehman, M. J. M. Séguret, T. Holland, E. P. Achterberg, A. Nabi and P. J. Worsfold, Analytica Chimica Acta, 2009, 652, 259-265.

20. A. Fan, Z. Cao, H. Li, M. Kai and J. Lu, Analytical Sciences, 2009, 25, 587-597.

21. J. I. Creamer and A. M. Buck, Luminescence, 2009, 24, 311-316.

22. T. G. Burdo and W. R. Seitz, Analytical Chemistry, 1975, 47, 1639-1643.

23. L. Li, M. A. Arnold and J. S. Dordick, Biotechnology and Bioengineering, 1993, 41, 11121120.

24. J. Lin, X. Shan, S. Hanaoka and M. Yamada, Lab Chip, 2001, 3, 5043-5051.

25. N. Feng, J. Lu, Y. He and J. Du, Luminescence, 2005, 20, 266-270.

26. G. E. Batley and D. Gardner, Estuarine and Coastal Marine Science, 1978, 7, 59-70.

27. O. Nozaki and H. Kawamoto, Luminescence, 2003, 18, 203-206.

28. O. Nozaki, M. Munesue and H. Kawamoto, Luminescence, 2007, 22, 401-406.

\section{Figures Caption}

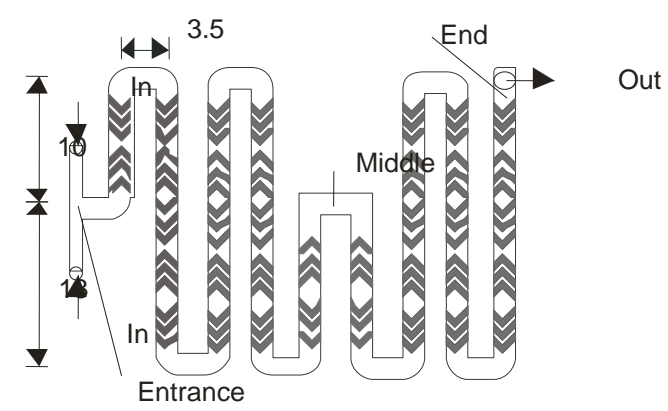

Figure 1. Schematic layout of microchip. Measurements are in millimetres (mm). 


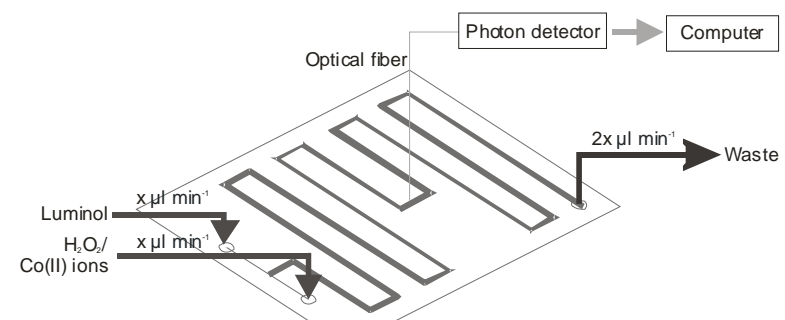

Figure 2. Experimental setup of the microchip system.

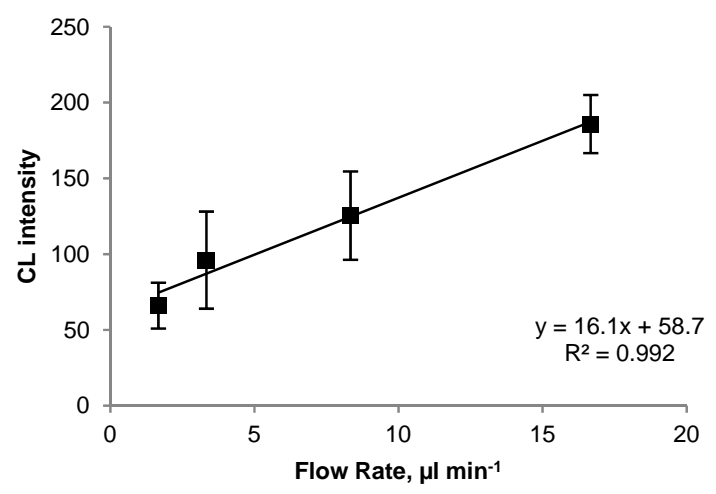

Figure 3. Effect of flow rate on $C L$ intensity at fixed position, $131 \mathrm{~mm}$ from the inlet. Error bars represent standard derivations.

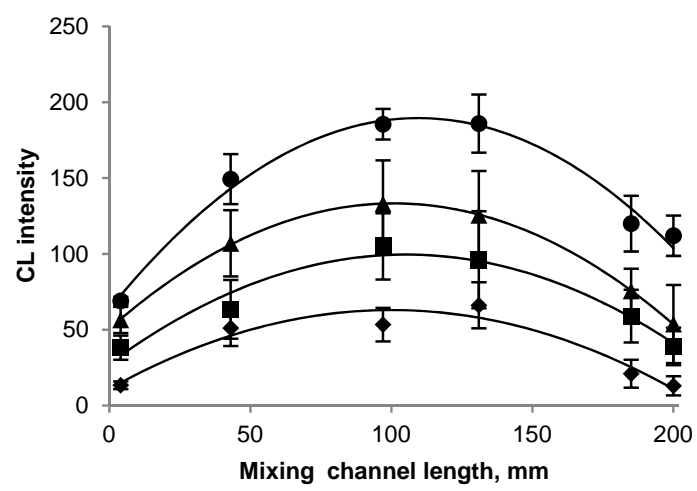

Figure 4. Effects of the mixing channel length on $C L$ intensity for different flow rate. The flow rates of samples and

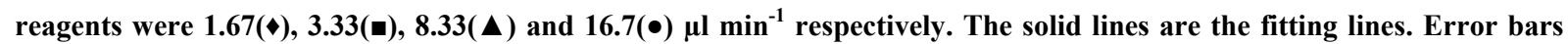
represent standard derivations. 


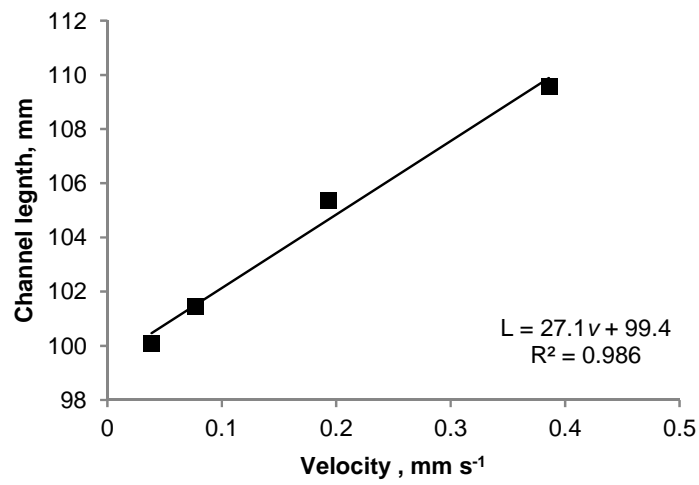

Figure 5. Effect of velocity in the channel on the position of the maximum CL intensity on the channel.

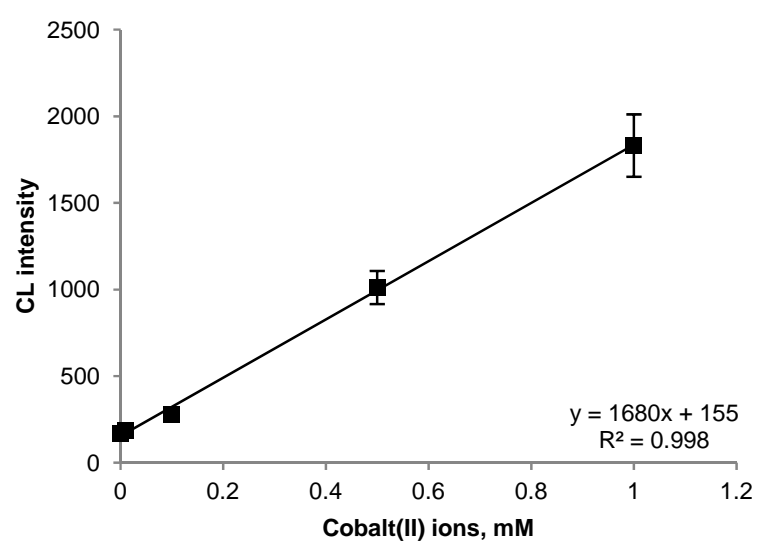

Figure 6. Effect of concentration of cobalt(II) ions on CL intensity. Error bars represent standard derivations. 


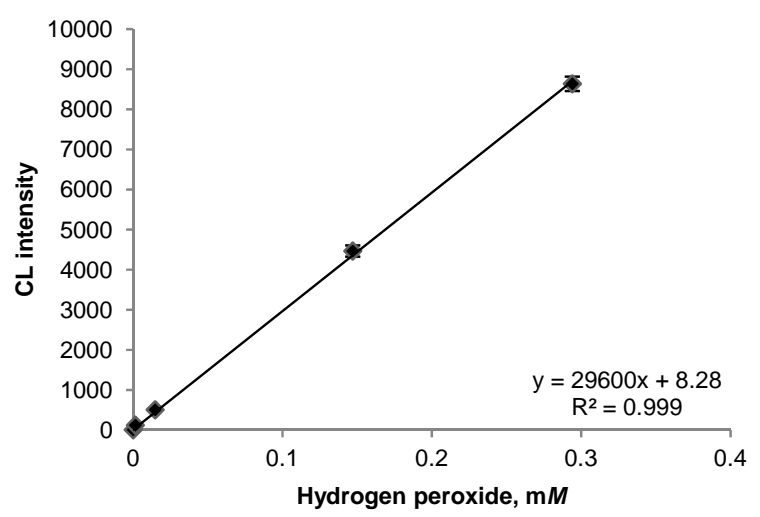

Figure 7. Effect of concentration of hydrogen peroxide on CL intensity. Error bars represent standard derivations. 